\title{
Design of the CMS upgraded trigger from Phase I to Phase II of the LHC
}

\author{
Silvio Donato*† \\ University of Zurich \\ E-mail: silvio.donato@cern.ch
}

The CMS experiment has a sophisticated two-level triggering system composed of the Level1, instrumented by custom-design hardware boards, and the High Level Trigger, a streamlined version of the offline reconstruction software running on a computer farm (more than 30k CPU cores). In 2017, the LHC delivered proton-proton collisions at a centre-of-mass energy of 13 $\mathrm{TeV}$ with a peak instantaneous luminosity larger than $2 \cdot 10^{34} \mathrm{~cm}^{-2} \mathrm{~s}^{-1}$, more than twice the peak luminosity reached during Run1 and far larger than the design value. The CMS Level-1 trigger was upgraded during the end-of-the year technical stop between 2015 and 2016 in order to improve its performance at high luminosity and at large number of simultaneous inelastic collisions per crossing (pile-up). All the electronic boards have been replaced and the upgraded electronics tested and commissioned with data. Smarter, more sophisticated, and innovative algorithms are now the core of the first decision layer of CMS: the upgraded trigger system implements pattern recognition and MVA (Boosted Decision Tree) regression techniques in the trigger boards for $\mathrm{p}_{\mathrm{T}}$ assignment, pile-up subtraction, and isolation requirements for electrons and tau leptons. In addition, the new global trigger is capable of evaluating complex selection algorithms such as those involving the invariant mass of trigger objects. The High Level Trigger features a trade-off between the offline complexity of the algorithms and the available computing power, and between the selection efficiency the maximum sustainable output rate. The trigger selections used in Run-2 will be presented, ranging from simpler single-object selections to more sophisticated algorithms combining different objects and applying analysis-level reconstruction and selection. This document describes the design and performance of the Phase I trigger and how it influences the path towards the Phase II upgrade necessary for the LHC run at a center-of mass energy of $14 \mathrm{TeV}$ with luminosity of $5-7 \cdot 10^{34} \mathrm{~cm}^{-2} \mathrm{~s}^{-1}$, corresponding to $140-200$ pile-up events. The addition of the tracker information at Level-1 and the enhanced computing resources at HLT will maintain the trigger efficiency at a similar level as the present one.

The 39th International Conference on High Energy Physics (ICHEP2018)

4-11 July, 2018

Seoul, Korea

${ }^{*}$ Speaker.

${ }^{\dagger}$ on behalf of the CMS collaboration. 


\section{Introduction}

The LHC instantaneous luminosity is steadily increasing. In the Run-2, it reached $\sim 2 \cdot 10^{34} \mathrm{~cm}^{-2} \mathrm{~s}^{-1}$, about two times the design value. The LHC will be upgraded to the High Luminosity LHC in 2026 to reach up to $7 \cdot 10^{34} \mathrm{~cm}^{-2} \mathrm{~s}^{-1}$ corresponding to 200 simultaneous protonproton collisions. The LHC experiments will install important upgrades during the Long Shutdown 3 (2024-26) to cope with such high luminosity. This document summarizes the main upgrades planned for the trigger system of the CMS experiment [1] and its expected performance.

\section{The upgraded CMS trigger system}

The trigger system of the CMS experiment is divided in two parts: the Level-1 trigger (L1) and the High Level Trigger (HLT). The L1 trigger uses a simplified event readout of the subdetectors and computes the trigger decision at $40 \mathrm{MHz}$ using customizable electronics. The upgraded L1 trigger [2] will have a larger output rate $(750 \mathrm{kHz}$ instead of $100 \mathrm{kHz})$ and a larger latency $(12.5 \mu \mathrm{s}$ instead of $4 \mu \mathrm{s}$ ) allowing to run more sophisticated triggers. The event readout of the L1 trigger will have a higher granularity and will include new detectors. The upgraded L1 trigger is composed of three levels. The first level is a simplified readout of the subdetectors, producing the first L1 objects (Trigger Primitive Generator, TPG). These objects are then combined in an intermediate step with, for instance, the reconstruction of the muon tracks. All information is then combined in the L1 correlator trigger to produce the final L1 objects (eg. jets and muons). Eventually, the L1 objects are used to evaluate the final trigger selection according to a L1 trigger menu. When an event is accepted by the L1 trigger, the full readout of all subdetectors takes place and the data are sent to the HLT.

The HLT farm is a computing farm that selects the events to be stored on disk. It uses algorithms that are similar to those used in the offline reconstruction, but in a simplified version to cope with the limited computing resources and the larger amount of events to be reconstructed. The upgraded HLT [3] will need to be able to run with the largest input rate and pileup. The HLT output rate is expected to increase from $1.0 \mathrm{kHz}$ to $7.5 \mathrm{kHz}$.

\section{Upgraded subdetectors and L1 trigger}

The CMS silicon tracker is going to be fully replaced [4]. The new outer tracker will be made of pairs of $1-4 \mathrm{~mm}$-distant modules. Each module will be able to reconstruct online at $40 \mathrm{MHz}$ the hit doublets compatible with tracks with $p_{T}$ larger than about $2 \mathrm{GeV}$ and to measure their transverse momentum.

The CMS endcap calorimeters will be replaced by a high-granularity calorimeter made of 52 silicon or scintillator layers. The L1 trigger will read the 14 electromagnetic and 24 hadronic layers with a granularity of $4 \mathrm{~cm}^{2}$. Given the huge amount of data, a zero suppression corresponding to 2 minimum ionizing particles will be applied. The trigger primitives generator will reconstruct the 2D energy deposit clusters in each sensitive layer and will link them into 3D clusters.

The upgraded L1 trigger will read the ECAL barrel calorimeter with a higher granularity (single crystal instead of $5 \times 5$ crystals). 
The muon detector will be upgraded using an improved version of RPC (iRPC) with a better time resolution (1.5 ns instead of $25 \mathrm{~ns}$ ), a better spatial resolution in DT, the addition of the new GEM detectors in the endcaps.

\section{Track-trigger improvements}

The addition of tracking in the L1 trigger will improve dramatically the trigger physics performance. The left side of Fig. 1 shows the improvement in the L1 trigger efficiency obtained using the L1 track (outer tracker) instead of the L1 muon (muon chambers) to measure the muon $p_{T}$.

L1 tracks allow also to distinguish between electrons and photons. The center of Fig. 1 shows the trigger efficiency as a function of the transverse energy threshold for a single electron, photon, and electron/photon trigger for the current and upgraded detector. Furthermore, L1 tracks allow to evaluate and cut on track isolation. On the right of Fig. 1 shows the rate reduction vs the signal efficiency for several track isolation cuts applied to a single photon trigger.
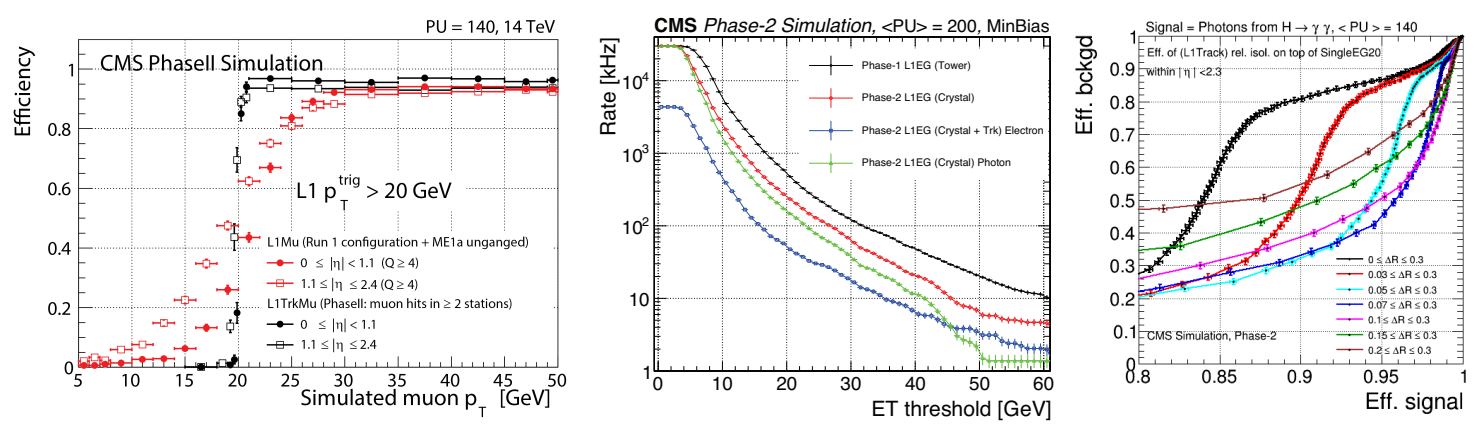

Figure 1: On the left, single muon trigger efficiency as a function of $p_{T}$ using L1 tracks (black) and L1 muons (red) [5]. On the center, single electron/photon trigger rate as a function of the transverse energy threshold [2]. On the right, background and signal efficiency for several track isolation cuts applied to a single photon trigger [5].

\section{Particle-flow and PUPPI (Pileup Per Particle Identification) at the L1 trigger}

The ultimate application of L1 tracks is to run the Particle Flow (PF) [6] and Pileup Per Particle Identification (PUPPI) [7] algorithms combining all subdetector data in order to get a better object reconstruction and mitigate the pileup effects.

The left side of Fig. 2 shows the rate vs efficiency for the missing transverse energy triggers with different thresholds for a semi-leptonic $t \bar{t}$ simulation. The right side of Fig. 2 shows the efficiency for different HT trigger with the same rate. Preliminary proof-of-principle to run PF and PUPPI in the L1 trigger boards have been successfully completed.

\section{High Level Trigger}

The computing power of the HLT farm will need to be increased of about a factor 20 for the High Luminosity LHC, assuming to keep a similar L1 and HLT trigger menu: a factor 7.5 is from 

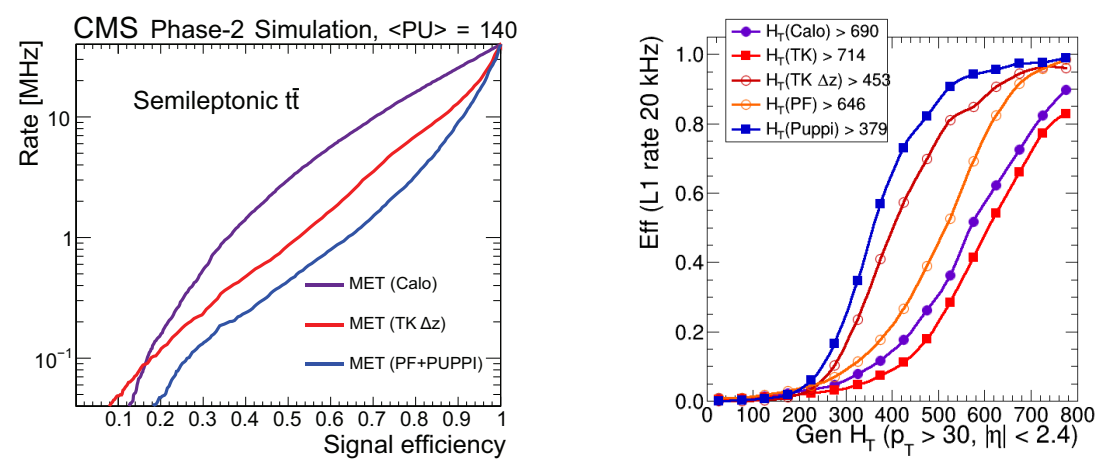

Figure 2: On the left, trigger efficiency for semileptonic $t \bar{t}$ events vs trigger rate for missing transverse energy triggers with different thresholds. On the right, efficiency for HT triggers as a function of the generator HT (right) at fixed rate. The plots have been obtained using the calorimetric variables, the tracks originating from the primary vertex, PF, or PF+PUPPI [2].

to the larger input rate and a factor 2.5 is due to the slower reconstruction of event with larger pileup. The DAQ bandwidth and the storage throughput are expected to increase similarly of about a factor 20.

In order to overcome such a big computing power request, alternative possibilities are being studied like the usage of heterogeneous architectures.

The rationale is to use coprocessors (eg. GPU or vectorizable CPU) to run some parts of the event reconstruction, for example tracking. coprocessors are installed in the event builder facility. A concrete example has been studied [8]: the usage of GPUs for pixel track seeding is 4.6 times faster than CPUs per cost unit and $+30 \%$ faster per electric power.

\section{References}

[1] S. Chatrchyan et al. [CMS Collaboration], JINST 3 (2008) S08004. doi:10.1088/1748-0221/3/08/S08004

[2] CMS Collaboration, CERN-LHCC-2017-013, CMS-TDR-017, https://cds.cern.ch/record/2283192.

[3] CMS Collaboration, CERN-LHCC-2017-014, CMS-TDR-018, https://cds.cern.ch/record/2283193.

[4] CMS Collaboration, CERN-LHCC-2017-009, CMS-TDR-014, https://cds.cern.ch/record/2272264.

[5] CMS Collaboration, CERN-LHCC-2015-010, LHCC-P-008, CMS-TDR-15-02, https://cds.cern.ch/record/2020886.

[6] A. M. Sirunyan et al. [CMS Collaboration], JINST 12 (2017) no.10, P10003 doi:10.1088/1748-0221/12/10/P10003 [arXiv:1706.04965 [physics.ins-det]].

[7] D. Bertolini, P. Harris, M. Low and N. Tran, JHEP 1410 (2014) 059 doi:10.1007/JHEP10(2014)059 [arXiv:1407.6013 [hep-ph]].

[8] F. Pantaleo, PhD thesis, University of Hamburg, CMS-TS-2017-028, https://cds.cern.ch/record/2293435. 